\title{
MAP-2 as an early marker of hippocampal damage after perinatal asphyxia and neuroprotective properties of Palmitoylethanolamide.
}

Francisco Capani ${ }^{1}$, Lucas Udovin ${ }^{1}$, Tamara Kobiec ${ }^{2}$, Camila Menendez $^{2}$, Toro-Urrego Nicolas ${ }^{1}$, Carlos Kusnier $^{1}$, Matilde Otero-Losada ${ }^{1}$ and Maria Ines Herrera ${ }^{2}$

${ }^{1}$ Universidad Abierta Interamericana (UAI) -CONICET, Buenos Aires, Buenos Aires, Argentina, ${ }^{2}$ Centro de Investigaciones en Psicología y Psicopedagogía (CIPP), Facultad de Psicología y Psicopedagogía, Universidad Católica Argentina (UCA), Buenos Aires, Argentina, Buenos Aires, Argentina

Statement of the Problem: Diminish in the oxygen levels prompted short and long-term alterations in synapses and related structures that are related to neuronal dysfunction and death. Perinatal asphyxia (PA) is an obstetric complication produced by an impaired gas exchange that lead to neonatal mortality and is a determinant factor for neurodevelopmental disorders $(1,2)$. Cumulative experimental evidence refers Palmitoylethanolamide (PEA) exerts neuroprotective actions in different models of brain injury and neurodegeneration (3) . Accordingly, we have observed PEA treatment could ameliorate hippocampal deficit in microtubule associated protein-2 (MAP-2) 1 month after PA. Therefore, the aim of the present study was to assess earlier neuroprotective effects of this endogenous compound using correlative light and electron microscopy.

Methodology \& Theoretical Orientation PA was induced by placing newborn Sprague Dawley rats in a 37 ${ }^{\circ} \mathrm{C}$ water bath for 19 minutes. PEA treatment $(10 \mathrm{mg} / \mathrm{kg})$ was administered subcutaneously during the first hour of life. Hippocampal modifications were analyzed by Immunohistochemistry and electron microscopy at postnatal day 21 (P21), once the animals had completed synapse formation and reflex maturation. In CA1 region, a decrease in MAP-2 reactive area was observed at P21 as a consequence of PA. In this way, MAP-2 appears as an early biomarker of PA-induced hippocampal damage (Fig 1). In addition, subcelular modifications were observed using electron microscopy techniques. After PA, neuronal cell body showed clear signs such as nuclear fragmentation, dark cytoplasm and vesicles accumulation. PEA reverted these modifications.

Conclusion \& Significance Therefore, PEA treatment could attenuate this hippocampal dendritic dysfunction, representing a putative neuroprotective agent for the developing injured brain. Future studies on long-term MAP-2 modifications might help determine the efficacy of PEA treatment on PA-induced dendritic cytoskeletal derangements.

Key words: perinatal asphyxia - MAP-2 - CA1 region - Palmitoylethanolamide - neuroprotection. 


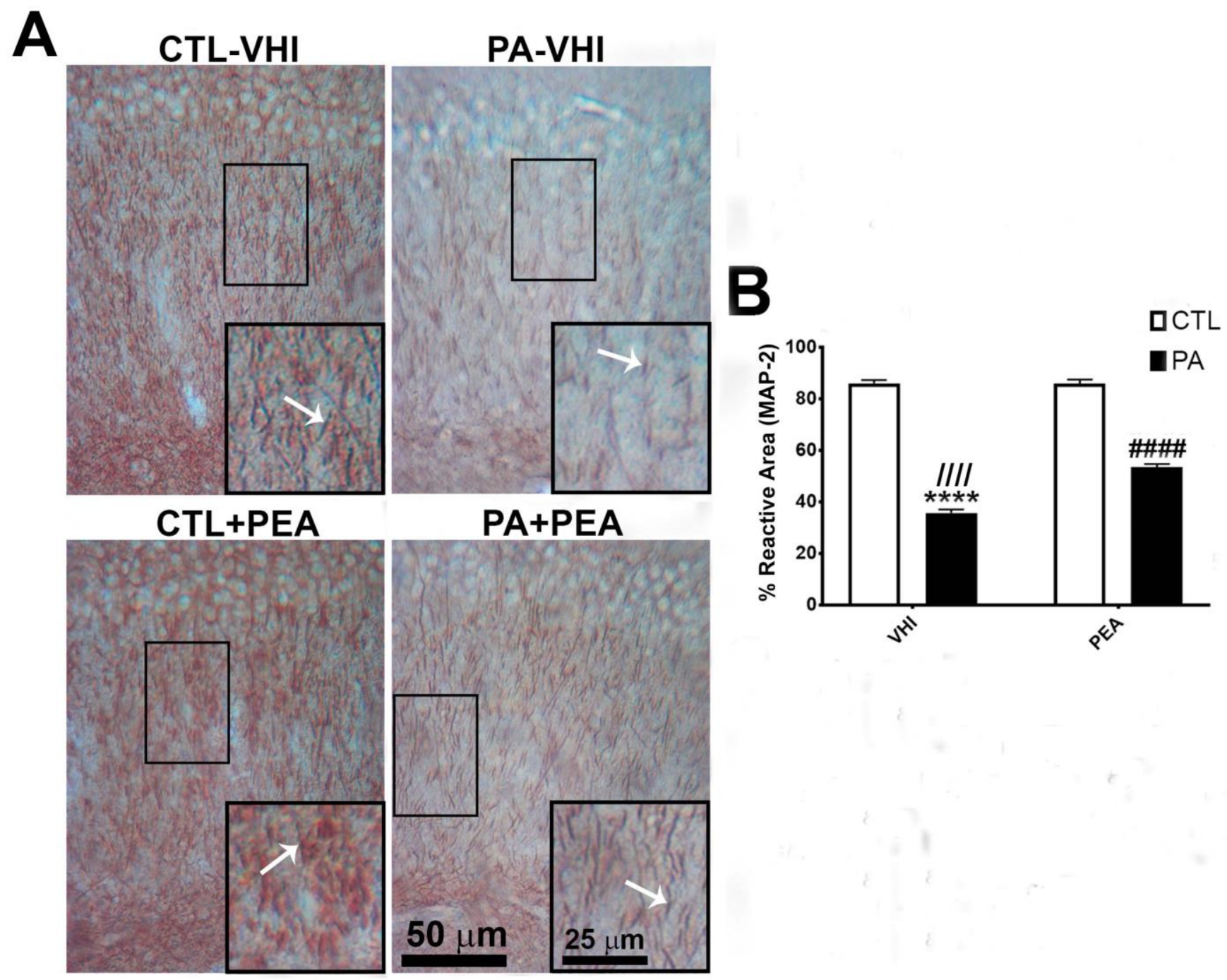

Figure 1. Microtubule-associated protein (MAP-2) immunostaining and protein expression levels in the rat hippocampus at P21. (A) Representative image of the stratum radiatum of CA1 hippocampal area immunostained for MAP-2 in the different groups. Scale bar: $50 \mu \mathrm{m}$. The main-image marked rectangular areas are shown magnified in the corresponding lower right margin. The white arrow indicates the positive immunostaining area for MAP-2. Scale bar: $25 \mu \mathrm{m}$. (B) Percentage of reactive area of MAP-2 positive dendrites. Bars and error bars represent mean \pm SEM. Statistical analyses were conducted by two-way ANOVA. Post hoc comparisons were adjusted by Bonferroni correction. $* * * * p<0.0001$, PA-VHI vs. CTLVHI; ////p < 0.0001, PA-VHI vs. PA+PEA; \#\#\#\# < 0.0001, PA+PEA vs. CTL-VHI. CTL-VHI, control rats treated with vehicle; PA-VHI, rats subjected to PA and treated with vehicle; PA+PEA, rats subjected to PA and PEA treatment; CTL+PEA, control rats treated with PEA.

\section{References}

1-Udovin L, Quarracino C, Herrera MI, Capani F, Otero Losada M, Perez-Lloret S (2020) Neural Plast. 2020 ;2020:1859431. doi: 10.1155/2020/1859431

2-Herrera MI, Kobiec T, Kölliker-Frers R, Otero-Losada M, Capani F (2020). Front Synaptic Neurosci. 2020 23;12:35. doi: 10.3389/fnsyn.2020.00035.

3-Herrera MI, Udovin L, Toro-Urrego N, Kusnier CF, Luaces JP, Capani F (2018) Front Neurosci. 28;12:145. doi: 10.3389/fnins.2018.00145 